\title{
A large international single-center case-series of coronary artery fistulas in children: When high clinical research standards adopted globally create exciting opportunities for the future
}

\author{
Petros V. Anagnostopoulos, MD, MBA
}

\footnotetext{
From the Division of Pediatric Cardiothoracic Surgery, American Family Children's Hospital, University of Wisconsin Hospital and Clinics, Madison, Wis.

Disclosures: Author has nothing to disclose with regard to commercial support.

Received for publication May 16, 2016; accepted for publication May 26, 2016; available ahead of print June 22, 2016.

Address for reprints: Petros V. Anagnostopoulos, MD, MBA, Division of Pediatric Cardiothoracic Surgery, American Family Children's Hospital, University of Wisconsin Hospital and Clinics, H4/358 Clinical Sciences Center, 600 Highland Ave, Madison, WI 53792 (E-mail: petros@ surgery.wisc.edu).

J Thorac Cardiovasc Surg 2016;152:1131-2

$0022-5223 / \$ 36.00$

Copyright (C) 2016 by The American Association for Thoracic Surgery

http://dx.doi.org/10.1016/j.jtcvs.2016.05.039
}

Isolated coronary artery fistulas (ICAFs) (Figure 1) are rare, representing $0.4 \%$ of congenital cardiac malformations. ${ }^{1,2}$ Most single-center series include a mix of pediatric and adult patients. $^{3-5}$ Experience with the management of ICAFs, however, has demonstrated that the clinical presentation, risk, and management options differ in adult and pediatric patients. ${ }^{2,4,6}$ Pediatric patients are diagnosed with asymptomatic murmurs, whereas older patients present with congestive heart failure, endocarditis, and myocardial infarction (MI). The risk of surgical repair in children is small. Adult perioperative morbidity and mortality risk are considerable as the result of early and late MI and coronary artery thrombosis. Transcatheter

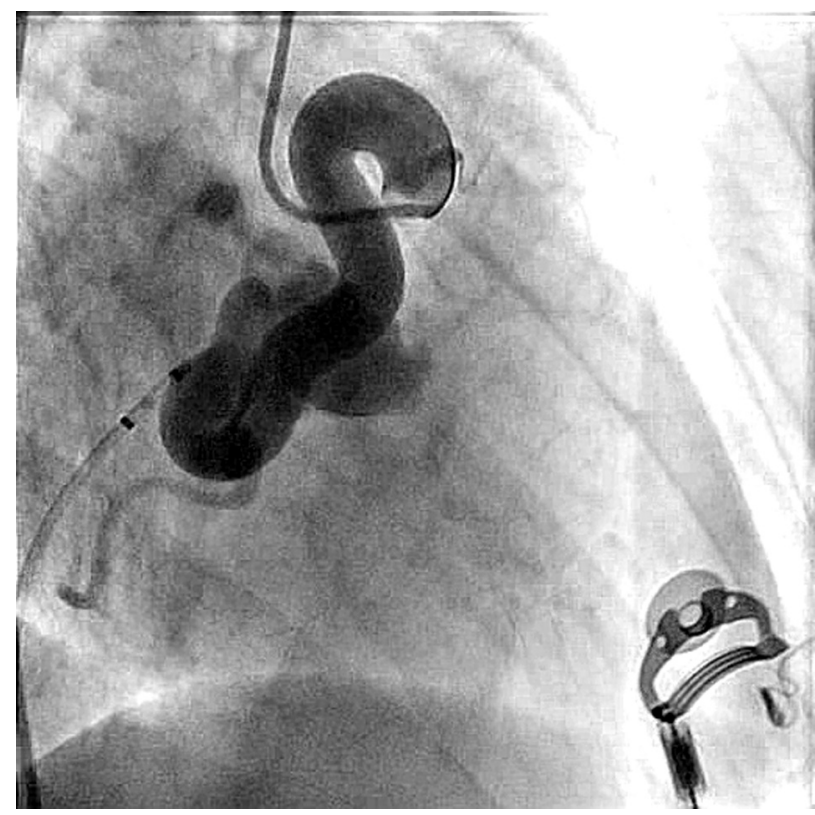

FIGURE 1. Angiogram of a large right coronary artery fistula.

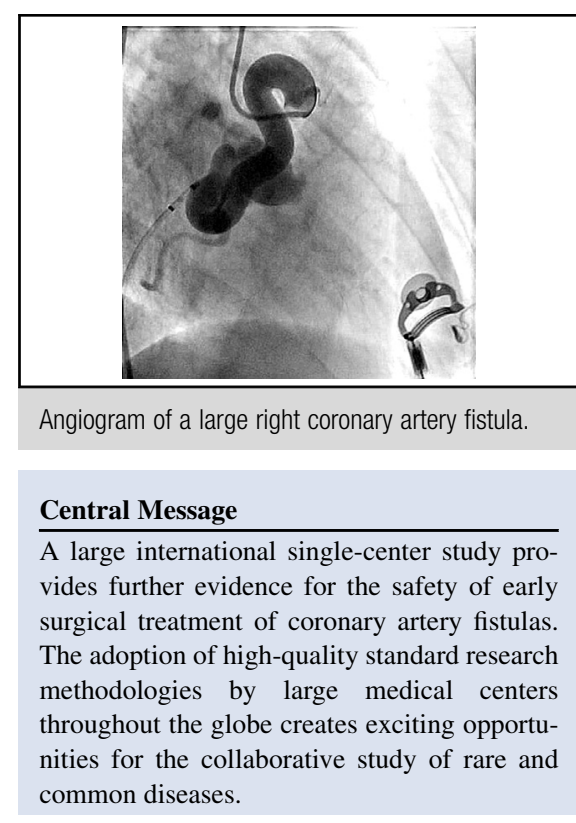

See Article page 1123 . therapies are attractive in adults but frequently not applicable to small children due to size and anatomy.

Although most agree that surgery is warranted in the symptomatic pediatric patient with ICAF, the indications and timing of intervention in asymptomatic patients remain controversial. ${ }^{6}$ Up to $39 \%$ of small coronary artery fistulas can spontaneously close, but if they do not, symptoms develop by the second decade and surgical risk increases. ${ }^{7,8}$ Even when surgery is performed successfully in young age, however, the risk of late MI does not disappear, especially if enlargement of the coronary artery proximal to the ICCAF persists.

In this issue of the Journal, Zhang and colleagues ${ }^{9}$ present the largest to date single-center series of pediatric patients with ICAF. The surgical results were excellent in 47 consecutive patients. There was no early or late mortality at a median follow-up of 7.8 years, and all patients were in New York Heart Association class I. Patients with intracardiac fistula repair had significantly less incidence of residual shunt ( $0 \%$ vs $32 \%$ for external or transcoronary ligation). This 
study provides further evidence that fistulas larger than $3 \mathrm{~mm}$ should be repaired early in childhood with minimal morbidity and mortality. The repair, if possible, should be intracardiac. We still do not know what percentage of patients will have regression of the dilatation of the coronary proximal to the fistula and in how many patients this dilatation will result in late thrombosis or embolization.

Conducting meaningful longitudinal studies in a patient cohort is laborious, difficult to accomplish, and requires incredible commitment of the care team, especially in the absence of multi-institutional databases like the ones in $\mathrm{Eu}-$ rope and North America. Zhang and colleagues ${ }^{9}$ are to be applauded. Their study includes a large number of patients followed closely by providers who analyzed the data carefully and presented them in a well-written paper. It is refreshing to see that high standards of clinical research have been adopted in centers all over the world and that the results of such research are shared in global platforms such as our Journal. This creates enormous potential for significant advancements in the study of common and rare diseases alike. One can only imagine what the impact for our patients would be if surgeons from different institutions and countries would take advantage of this common research culture and collaboratively tackled the many remaining unanswered questions of our specialty.

\section{References}

1. Mangukia CV. Coronary artery fistula. Ann Thorac Surg. 2012;93:2084-92.

2. Mavroudis C, Backer CL, Rocchini AP, Muster AJ, Gevitz M. Coronary artery fistulas in infants and children: a surgical review and discussion of coil embolization. Ann Thorac Surg. 1997;63:1235-42.

3. Gowda ST, Latson LA, Kutty S, Prieto LR. Intermediate to long-term outcome following congenital coronary artery fistulae closure with focus on thrombus formation. Am J Cardiol. 2011;107:302-8.

4. Said SM, Burkhart HM, Schaff HV, Connolly HM, Phillips SD, Suri RM, et al. Late outcome of repair of congenital coronary artery fistulas - a word of caution. J Thorac Cardiovasc Surg. 2013;145:455-60.

5. Kamiya H, Yasuda T, Nagamine H, Sakakibara N, Nishida S, Kawasuji M, et al. Surgical treatment of congenital coronary artery fistulas: 27 years' experience and a review of the literature. J Card Surg. 2002;17:173-7.

6. Yim D, Yong MS, d'Udekem Y, Brizard CP, Konstantinov IE. Early surgical repair of the coronary artery fistulae in children: 30 years of experience. Ann Thorac Surg. 2015;100:188-94.

7. Welisch E, Norozi K, Burrill L, Rauch R. Small coronary artery fistulae in childhood: a 6-year experience of 31 cases in a tertiary paediatric cardiac centre. Cardiol Young. 2016;26:738-42.

8. Valente AM, Lock JE, Gauvreau K, Rodriguez-Huertas E, Joyce C, Armsby L, et al. Predictors of long-term adverse outcomes in patients with congenital coronary artery fistulae. Circ Cardiovasc Interv. 2010;3:134-9.

9. Zhang W, Hu R, Zhang L, Zhu H, Zhang H. Outcomes of surgical repair of pediatric coronary artery fistulas. J Thorac Cardiovasc Surg. 2016;152:1123-30.e1. 